\title{
The Meissner Effect and the Physics of Light
}

\author{
Ana Paula Lima \\ CBPF - Centro Brasileiro de Pesquisas Físicas \\ E-mail: ana_crlima@hotmail.com
}

\section{Edgar Marcelino de Carvalho Neto*}

CBPF - Centro Brasileiro de Pesquisas Físicas

E-mail: edgarufbaegmail.com

\section{José Chauca}

CBPF - Centro Brasileiro de Pesquisas Físicas

E-mail: jlchmecbpf.br

\section{Renato Doria}

AprendaNet, Brazil

E-mail: doriaeaprendanet.com.br

\section{Inacio Washington}

AprendaNet, Brazil

E-mail: w.inaciodhotmail.com

\begin{abstract}
A new approach for electromagnetism comes from the so-called Physics of Light. Based on a new universalism for light invariance, it generates an electromagnetism beyond the electric charge. As a consequence, it brings new electromagnetic fields different from the usual pair.

Thus this works studies the possibilities of electromagnetic fields originated from the approach set out by the Physics of Light. A massive photon comes out without violating gauge invariance. Considering a model with just two fields $\left\{A_{\mu}, B_{v}\right\}$ where $A_{\mu}$ is the usual photon and $B_{v}$ is the massive photon, one derives granular and collective electromagnetic fields. There is a new possibility for explaining the Meissner effect different from Procca in the framework of Physics of Light.
\end{abstract}

4th International International Conference on Fundamental Interactions August 1 - 7, 2010

Viçosa, Brazil

\footnotetext{
* Poster Section
} 


\section{Introduction}

A new gauge approach means to consider a set of fields $\left\{A_{\mu I}\right\}$ transforming under a same group. Quark confinement and complexity are supporting this antireductionist aproach to physics. They are saying that one should look beyond the parts for the phenomena interpretation [1].

Thus there are strong motivations for describing physics through an antireductionist gauge model. Nature is more than its parts. This fact suggest us to introduce a common group of fields $\left\{A_{\mu I}\right\}$ transforming under a same gauge parameter as

$$
A_{\mu I} \rightarrow A_{\mu I}=A_{\mu I}^{\prime}+\partial_{\mu} P_{I}(\alpha)
$$

where $I$ means a diversity index which varies from $I=1, \ldots, N$ and $P_{I}(\alpha)$ a gauge parameter polynomial expansion, $P_{I}(\alpha)=a_{I m} \alpha^{m}(x)$ [2].

Eq. (1.1) leads to the Lagrangian

$$
L=Z_{\mu v} Z^{\mu v}-\frac{1}{2} m_{I J}^{2} A_{\mu}^{I} A^{\mu J}
$$

which is gauge invariant under the condition $a_{I} P^{\prime I}(\alpha)=0$. Its correspondent fields strength are

$$
Z_{[\mu v]}=a_{I} F_{[\mu v]}^{I}+z_{[\mu v]}
$$

and

$$
Z_{(\mu v)}=b_{I} S_{(\mu v)}^{I}+z_{(\mu v)}+g_{\mu v}\left(c_{I} S_{\alpha}^{\alpha I}+z_{\alpha}^{\alpha}\right)
$$

where

$$
\begin{gathered}
F_{\mu v I}=\partial_{\mu} A_{v I}-\partial_{v} A_{\mu I}, \quad S_{\mu v I}=\partial_{\mu} A_{v I}+\partial_{v} A_{\mu I} \\
z_{[\mu v]}=\gamma_{[I J]} A_{\mu}^{I} A_{v}^{J}, \quad z_{(\mu v)}=\gamma_{(I J)} A_{\mu}^{I} A_{v}^{J}
\end{gathered}
$$

Notice that while eq. (1.5) contains a granular composition of fields, eq. (1.6) propiates a collective term.

Calculating the corresponding field equations, one gets

$$
\partial_{v} T_{I}^{v \mu}+m_{(I J)}^{2} A^{\mu J}=J_{I}^{\mu}
$$

where

$$
T_{I}^{v \mu}=\left(a_{I} a_{J}+b_{I} b_{J}\right) F^{v \mu J}+a_{I} z^{[v \mu]}+b_{I} z^{(v \mu)}+\left(b_{I}+5 c_{I}\right) z_{\alpha}^{\alpha}
$$

and

$$
J_{I}^{\mu}=\gamma_{[I J]} Z^{[\mu}{ }_{v]} A^{v J}+\gamma_{(I J)} Z^{(\mu}{ }_{v)} A^{v J}+\gamma_{(I J)} Z_{\alpha}^{\alpha} A^{\mu J}
$$

In order to simplify eq. (1.7), a gauge fixing term $\xi_{I J} \partial_{\alpha} A^{\alpha I} \partial_{\beta} A^{\beta J}$ and the kinetic identity $\partial_{v} S_{I}^{\nu \mu}=\partial_{v} F_{I}^{v \mu}+\eta^{v \mu} \partial_{\nu} S_{\alpha}^{\alpha I}$ were introduced. 
A second type of equations for analysing this abelian model are the Bianchi identities. They are

$$
\begin{gathered}
\partial_{\mu} F_{v \rho I}+\partial_{\rho} F_{\mu v I}+\partial_{v} F_{\rho \mu I}=0 \\
\partial_{\mu} z_{[v \rho]}+\partial_{v} z_{[\rho \mu]}+\partial_{\rho} z_{[\mu v]} \\
=\gamma_{[I J]} A_{v}^{I} F_{\mu \rho}^{J}+\gamma_{[I J]} A_{\rho}^{I} F_{v \mu}^{J}+\gamma_{[I J]} A_{\mu}^{I} F_{\rho v}^{J}
\end{gathered}
$$

and

$$
\begin{array}{r}
\partial_{\mu} z_{(v \rho)}+\partial_{v} z_{(\rho \mu)}+\partial_{\rho} z_{(\mu v)} \\
=\gamma_{(I J)} A_{\mu}^{I} S_{v \rho}^{J}+\gamma_{(I J)} A_{v}^{I} S_{\rho \mu}^{J}+\gamma_{(I J)} A_{\rho}^{I} S_{\mu v}^{J}
\end{array}
$$

Here, we should mention that our formulation though it sets out a non-linear extension of the electromagnetic theory, it does not correspond to any particular regime of the Born-Infeld action. We extend Electromagnetism by introducing extra gauge potentials and non-linearity comes out as a consequence of gauge symmetry.

\section{Global Maxwell Equation}

Expressing the previous equations in vector form, $A_{I}^{\mu} \equiv\left(\phi_{I}, \vec{A}_{I}\right)$, ones derives the so-called Global Maxwell equation

$$
\begin{array}{r}
\vec{\nabla} \cdot\left(\vec{E}_{I}+b_{I} \vec{e}\right)+m_{I}^{2} \phi_{I}=\rho_{I}(A) \\
\vec{\nabla} \times\left(\vec{B}_{I}+b_{I} \vec{b}\right)+\frac{\partial}{\partial t}\left(\vec{E}_{I}+b_{I} \vec{e}\right)+m_{I}^{2} \vec{A}_{I}=\vec{J}_{I}(A) \\
\vec{\nabla} \times \vec{E}_{I}+\frac{\partial \vec{B}_{I}}{\partial t}=0 \\
\vec{\nabla} \cdot \vec{B}_{I}=0 \\
\vec{\nabla} \times \vec{e}+\frac{\partial \vec{b}}{\partial t}=\gamma_{[I J]}\left(\vec{A}^{I} \times \vec{E}^{J}-\phi^{I} \vec{B}^{J}\right) \\
\vec{\nabla} \cdot \vec{b}=\gamma_{[I J]} \vec{A}^{I} \cdot \vec{B}^{J}
\end{array}
$$

where

$$
\begin{array}{r}
\vec{E}_{I}=-\vec{\nabla} \phi_{I}-\frac{\partial \vec{A}_{I}}{\partial t}, \quad \vec{B}_{I}=\vec{\nabla} \times \vec{A}_{I} \quad \text { are the usual electromagnetic fields } \\
\vec{e}=-\gamma_{[J K]} \phi^{J} \vec{A}^{K}, \quad \vec{b}=\gamma_{[J K]} \vec{A}^{J} \times \vec{A}^{K} \quad \text { are the collective fields }
\end{array}
$$

Similarly, one gets longitudinal electromagnetic fields derived from tensor $S_{\mu \nu I}\left(\varepsilon \rightarrow \varepsilon, \beta_{i j}\right)$ and the collective fields derived from $z_{\mu v}\left(s, \vec{s}, s_{i j}\right)$. For simplification, the sources were written just symbolically. The symmetric fields are there. 


\section{Two Fields Solution}

In order to solve the above Global Maxwell equation one has to rewrite it in terms of potential fields $\left(\phi_{I}, \vec{A}_{I}\right)$. It gives,

$$
\begin{array}{r}
a_{I J} \nabla^{2} \phi^{K}+b_{I J K} \vec{A}^{K} \cdot \vec{\nabla} \phi^{J}+c_{I J K} \phi^{J} \vec{\nabla} \cdot \vec{A}^{K} \\
+d_{I J K} \vec{A}^{J} \cdot \frac{\partial \vec{A}^{K}}{\partial t}+c_{I J K} \phi^{J} \frac{\partial \phi^{K}}{\partial t}+f_{I K} \frac{\partial^{2} \phi^{K}}{\partial t^{2}}-m_{(I K)}^{2} \phi^{K} \\
=g_{I J K L} \phi^{J} \phi^{K} \phi^{L}+h_{I J K L} \phi^{K} \vec{A}^{J} \cdot \vec{A}^{L}
\end{array}
$$

and

$$
\begin{array}{r}
a_{I K} \nabla^{2} \vec{A}^{K}-a_{I K} \vec{\nabla}\left(\vec{\nabla} \cdot \vec{A}^{K}\right)+i_{I J K}\left(\vec{A}^{J} \cdot \vec{\nabla}\right) \vec{A}^{K} \\
+j_{I J K} \vec{A}^{J}\left(\vec{\nabla} \cdot \vec{A}^{K}\right)+l_{I J K}\left(\vec{\nabla} \cdot \vec{A}^{K}\right) \vec{A}^{J}+m_{I J K} \phi^{J} \vec{\nabla} \phi^{K} \\
+a_{I K} \frac{\partial^{2} \vec{A}^{K}}{\partial t^{2}}+a_{I K} \vec{\nabla} \frac{\partial \phi^{K}}{\partial t}+p_{I J K} \phi^{J} \frac{\partial \vec{A}^{K}}{\partial t}+m_{(I K)}^{2} \vec{A}^{K} \\
=n_{I J K L} \phi^{J} \phi^{K} \vec{A}^{L}+o_{I J K L}\left(\vec{A}^{K} \cdot \vec{A}^{L}\right) \vec{A}^{J}
\end{array}
$$

where $a_{I J}, \ldots o_{I J K L}$ are free coefficients which can take any value without breaking gauge invariance. They are functions on the initial parameters stipulated through eqs. (1.3-1.6).

Considering the two fields case $\left\{A_{\mu}, B_{v}\right\}$ where $A_{\mu} \equiv(\phi, \vec{A}), B_{\mu}=(\varphi, \vec{B})$ and taking the static approximation, Coulomb gauge $\phi=0, \vec{\nabla} \cdot \vec{A}=0$ and spherical symmetry, one derives from eqs. (3.1-3.2) the following four equations

$$
\begin{array}{r}
a_{12} \nabla^{2} \varphi+b_{121} \vec{A} \cdot \vec{\nabla} \varphi+b_{122} \vec{B} \cdot \vec{\nabla} \varphi+c_{122} \varphi \vec{\nabla} \cdot \vec{B}-m_{(12)}^{2} \varphi \\
=g_{1222}(\varphi)^{3}+h_{1121} \varphi(\vec{A} \cdot \vec{A})+\left(h_{1122}+h_{1221}\right) \varphi \vec{A} \cdot \vec{B}+h_{1222} \varphi \vec{B} \cdot \vec{B} \\
a_{22} \nabla^{2} \varphi+b_{221} \vec{A} \cdot \vec{\nabla} \varphi+b_{222} \vec{B} \cdot \vec{\nabla} \varphi+c_{222} \varphi \vec{\nabla} \cdot \vec{B}-m_{(22)}^{2} \varphi \\
=g_{2222}(\varphi)^{3}+g_{2121} \varphi \vec{A} \cdot \vec{A}+\left(g_{2122}+g_{2221}\right) \varphi \vec{A} \cdot \vec{B}+g_{2222} \varphi(\vec{B} \cdot \vec{B}) \\
a_{12} \nabla^{2} \vec{B}-a_{12} \vec{\nabla}(\vec{\nabla} \cdot \vec{B})+i_{111}(\vec{A} \cdot \vec{\nabla}) \vec{A}+i_{112}(\vec{A} \cdot \vec{\nabla}) \vec{B} \\
+i_{121}(\vec{B} \cdot \vec{\nabla}) \vec{A}+i_{122}(\vec{B} \cdot \vec{\nabla}) \vec{B}+j_{112} \vec{A}(\vec{\nabla} \cdot \vec{B})+j_{122} \vec{B}(\vec{\nabla} \cdot \vec{B}) \\
+l_{111}(\vec{\nabla} \cdot \vec{A}) \vec{A}+l_{112}(\vec{\nabla} \cdot \vec{B}) \vec{A}+l_{121}(\vec{\nabla} \cdot \vec{A}) \vec{B}+l_{122}(\vec{\nabla} \cdot \vec{B}) \vec{B} \\
+m_{122} \varphi \vec{\nabla} \varphi+m_{(11)}^{2} \vec{A}+m_{(12)}^{2} \vec{B} \\
=n_{1221}(\varphi)^{2} \vec{A}+n_{1222}(\varphi)^{2} \vec{B}+o_{1111}(\vec{A} \cdot \vec{A}) \vec{A} \\
+\left(o_{1112}+o_{1121}\right)(\vec{A} \cdot \vec{B}) \vec{A}+o_{1122}(\vec{B} \cdot \vec{B}) \vec{A}+o_{1211}(\vec{A} \cdot \vec{A}) \vec{B} \\
+\left(o_{1212}+o_{1221}\right)(\vec{A} \cdot \vec{B}) \vec{B}+o_{1222}(\vec{B} \cdot \vec{B}) \vec{B} \\
+i_{221}(\vec{B} \cdot \vec{\nabla}) \vec{A}+i_{222}(\vec{B} \cdot \vec{\nabla}) \vec{B}+j_{212} \vec{A}(\vec{\nabla} \cdot \vec{B})+j_{222} \vec{B}(\vec{\nabla} \cdot \vec{B})
\end{array}
$$




$$
\begin{array}{r}
+l_{211}(\vec{\nabla} \cdot \vec{A}) \vec{A}+l_{212}(\vec{\nabla} \cdot \vec{B}) \vec{A}+l_{221}(\vec{\nabla} \cdot \vec{A}) \vec{B}+l_{222}(\vec{\nabla} \cdot \vec{B}) \vec{B} \\
+m_{222} \varphi \vec{\nabla} \varphi+m_{(21)}^{2} \vec{A}+m_{(22)}^{2} \vec{B} \\
=n_{2221}(\varphi)^{2} \vec{A}+n_{2222}(\varphi)^{2} \vec{B}+o_{2111}(\vec{A} \cdot \vec{A}) \vec{A} \\
+\left(o_{2112}+o_{2121}\right)(\vec{A} \cdot \vec{B}) \vec{A}+o_{2122}(\vec{B} \cdot \vec{B}) \vec{A}+o_{2211}(\vec{A} \cdot \vec{A}) \vec{B} \\
+\left(o_{2212}+o_{2221}\right)(\vec{A} \cdot \vec{B}) \vec{B}+o_{2222}(\vec{B} \cdot \vec{B}) \vec{B}
\end{array}
$$

with a solution without considering sources

$$
\begin{gathered}
\varphi(r)=\frac{\varphi_{0} e^{-\beta r}}{r}, \quad \beta=\sqrt{\frac{m_{(12)}^{2} b_{221}-m_{(22)}^{2} b_{121}}{a_{12} b_{221}-a_{22} b_{121}}} \\
B(r)=\frac{B_{0} e^{-\alpha r}}{r^{2}} \\
\alpha=\sqrt{\left(\frac{b_{122} b_{221}}{b_{221} c_{122}-b_{121} c_{222}}\right)\left(\frac{m_{(12)}^{2} b_{221}-m_{(22)}^{2} b_{121}}{a_{12} b_{221}-a_{22} b_{121}}\right)} \\
A(r)=\frac{a}{r+\gamma}+\frac{b}{r(r+\gamma)}+\frac{c \cdot r}{r+\gamma}+\left(\frac{e}{r}+f\right) \frac{1}{r(r+\gamma)} e^{-\alpha r}
\end{gathered}
$$

which yields the following spectrum of electromagnetic fields

$$
\begin{gathered}
\vec{E}_{1}=0, \quad \vec{E}_{2}=\frac{\phi_{0} \beta}{r} e^{-\beta r}\left(1+\frac{1}{r}\right) \widehat{r} \\
\vec{B}_{1}=0, \quad \vec{B}_{2}=0 \\
\varepsilon_{1}=0, \quad \varepsilon_{2}=0, \quad \vec{\varepsilon}_{1}=0, \quad \vec{\varepsilon}_{2}=-\frac{\phi_{0} \beta e^{-\beta r}}{r}\left(1+\frac{1}{r}\right) \widehat{r} \\
s=\frac{\gamma_{[22]} \phi_{0}^{2} e^{-2 \beta r}}{r^{2}}, \quad \vec{s}=\left[\frac{\gamma_{[21]} \phi_{0} e^{-\beta r}}{r} A(r) \widehat{r}, \quad \vec{b}=0\right. \\
\beta_{(21)} \phi_{0} e^{-\beta r} \\
r
\end{gathered}
$$

\section{Conclusion}

Thus, due to the simplification on the spherical symmetry, the magnetic field presence nearly disappear, unless for $\beta_{i 2}^{i}$, but through to show a Meissner performance. On the other hand, the Meissner damping effect [3] can be more clearly noticed through the electric fields. It is interesting to observe that cut-off parameters as $\alpha$ and $\beta$ do not depend only of the mass. In a further work we intend to investigate the influence coming from the interactive sector. 


\section{References}

[1] 50 years of Yang-Mills, edited by Gerardus't Hooft, World Scientific, 2005.

[2] Dias, S. A., Doria, R. M.; Valle, J. L. Matheus., Rev. Bras. Fis, vol.21, num.1, 1991; C.A.S. Almeida and R. M. Doria, Rev. Bras. Fis, vol 13, num.21, 1991; N. Chair, J. A. Helayël-Neto and William Smith, Phys. Lett. B 233, 1989; C. M. Doria, R. M. Doria, F. A. B. Rabelo de Carvalho, Acta Physica Hungarica 73, 51-58, 1993.

[3] S. Stemberg “On the London Equations”, Physics 89, PNAS USA, 1992. 